\title{
Spectral Efficiency and User Diversity Gains Through Cooperative Fixed Relays
}

\author{
A. Adinoyi and H. Yanikomeroglu \\ Broadband Communications and Wireless Systems (BCWS) Centre \\ Dept. of Systems and Computer Engineering \\ Carleton University, Ottawa, Canada
}

\begin{abstract}
Cooperative diversity through mobile terminals is faced with a number of deployment challenges such as security (as terminals have to detect partner's signals), incentive and coercion packages (as users have to be motivated to use their terminals to assist others), and huge capital investment (as terminals have to be modified to make them ready for cooperation). This paper discusses fixed relay-enabled user cooperation that are being considered in the WINNER project for deploying beyond 3G wireless communication networks. The schemes operate by engaging two users in cooperation without their knowledge. The schemes which can be viewed as an add-on to network facility have significant impact on the costs, security and end-to-end (E2E) performance of two-hop wireless networks. In addition, the proposed schemes can be used in a number of networks since current terminals do not require any modifications.
\end{abstract}

\section{INTRODUCTION}

The explicit user cooperation diversity schemes discussed in [1], [2] require that at least two users are in the network and they are willing to cooperate. To sustain such a cooperation, coercion or incentives for the cooperating partners might be needed. In the conceptual presentation of user cooperation diversity, it is also noted that the two cooperating partners achieve mutual instant gain when both users face statistically similar channels to destination. However, in dissimilar channels, the gain for the user that has a better channel is not that instant. In this situation, a "greedy" partner might question the need for cooperation when it faces a good channel to destination. Furthermore, explicit cooperation requires that each cooperating partner detects the data of the other user. Hence, the data of each user would need to be protected from "a malicious" partner which adds another dimension to the security challenges of wireless networks.

From economic point of view, explicit cooperation requires that terminals are modified in order to be able to perform their new tasks, which leads to an increase in terminal complexities. Given that hundreds to thousands of wireless terminals are often served in a network, this involves significant capital investment, thereby imposing capital burden on both the service providers and end users.

To circumvent the aforementioned problems of userdependent cooperative diversity techniques, this paper discusses fixed relay-enabled user cooperation. The cooperation

This work was supported in part by the Natural Sciences \& Engineering Research Council of Canada (NSERC) under participation in project WINNER (Wireless World Initiative New Radio) - www.ist-winner.org. exploits the existing infrastructure-based relays which service providers deploy for the main purpose of coverage extension [3]. The scheme operates as follows: two users, ignorant of their cooperation, are engaged in it through two fixed relays. The user terminals do not need to detect each other's data. The benefits derived from this type of cooperation are obvious. First, the privacy of users is not compromised and secondly, sanctioning or rewarding the users for participating in the cooperation is not required as the cooperation is transparent to the users. In addition to these benefits, the fixed relay-enabled user cooperation ensures that user terminals are simple, routing is simple, and system deployment is flexible. These are the features that any service provider would like to maintain an unfettered control on.

A typical example of the flexibility of the proposed schemes is the ease with which it can support heterogeneous mode of relaying. It is important to note that most of the previous cooperative relaying schemes faces stiff challenges to operate on two air interfaces since the first and second hops are inherently coupled. This situation is different in the proposed scheme, where the two hops are decoupled. Therefore, the proposed cooperative techniques can easily be adapted to support heterogeneous relaying (i.e., two different air interfaces for the relay's receptions and transmissions can be used, in contrast to single air interface in homogeneous relaying). This, for example, implies that the receptions of both the relay and base station (BS) could be on two different frequency bands. One of these bands could be an unlicensed frequency band.

In Sec. II, the description of the proposed schemes is given and some realization examples are discussed. Then, an analytical framework for evaluating the system performance is examined in Sec. III. Numerical examples are given in Sec. IV and conclusions are drawn in Sec. V.

\section{SYSTEM DESCRIPTIONS}

The signal processing at the relays for the proposed schemes are classified into two - spatial division multiple access (SDMA)-based and multiuser detection (MUD)-based processing. In the multi-antenna relay architecture, the relays use the principle of SDMA for the detection of a single user (Fig. 1). However, in a single antenna relay architecture, the multiuser detection (MUD) capability is required as shown in a generic form (Fig. 2) where it is assumed that the users are assigned some spreading (orthogonal) codes. 


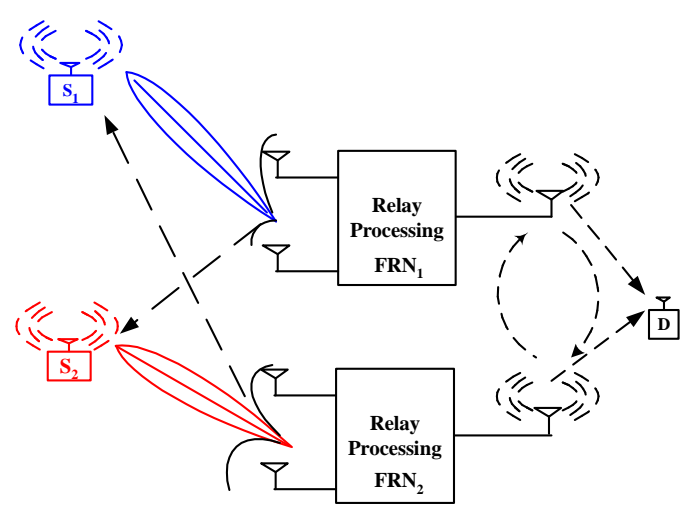

(a)

\begin{tabular}{|c|c|c|}
\hline $\begin{array}{l}\mathrm{S}_{1}\left(\mathrm{~d}_{1}\right) \ldots>\mathrm{FRN}_{1} \\
\mathrm{~S}_{2}\left(\mathrm{~d}_{2}\right) \ldots \mathrm{FRN}_{2}\end{array}$ & \begin{tabular}{|l}
$F R N_{1}\left(d_{1}\right) \ldots>D$ and $F R N_{2}$ \\
$F R N_{2}\left(d_{2}\right) \ldots>D$ and $F R N_{1}$
\end{tabular} & $\begin{array}{l}F R N_{1}\left(d_{2}\right) \ldots>D \\
F R N_{2}\left(d_{1}\right) \ldots>D\end{array}$ \\
\hline
\end{tabular}

(b)

Fig. 1. Fixed relay-enabled user cooperation: Realization I.

In general, after the relays detect the signal in the first hop, they could engage into cooperation using, for example, distributed space-time coding (DSTC)[4]. The scheme using SDMA called realization I operates in the following manners. Each relay detects the signal of one user by directing a null towards the other (Fig. 1 (a)). To engage the data of the two users in the distributed space-time coding, the signal of the nulled user is obtained by the other relay node by exploiting the broadcast nature of wireless channels. This exchange of user signals is accomplished in the second time slot when each relay node transmits to destination (Fig. 1 (b)). This imposes full duplex mode of operation on the relay node which has stringent practical implications. The three-slot protocol combines two-hop relaying technique with a (virtual) two transmit antenna scheme.

The second scheme referred to as realization II (Fig. 2) operates in the following way. Multi-user detection method is used at each relay for detecting the signals of the two partners. The MUD and some form of spreading are necessary for the single antenna relay architecture. For realization II the system could operate in a "pre-determined" distributed processing mode (e.g., space-time coding), the relays in this case, do not need to exchange any instantaneous information as the cooperation is agreed upon a priori. One advantage of this is the removal of the full-duplex requirement on the relays. In this mode, each relay assumes that the signals of the users it detects, have the same reliability as those detected by the cooperating relay. They simply employ these signals to realize the distributed space time coding. In the implementation of this scheme, however, automatic repeat request (ARQ) in conjunction with cyclic redundancy check (CRC) might be required to alleviate the problem of performing distributed signal processing with data/signals that are detected in error. Table I describes the distributed transmissions accomplished by coding across time and space. The datum of user $k$ detected at relay station $i$ is denoted as $d_{k}^{(i)}$.

For fair comparison with reference schemes, the following

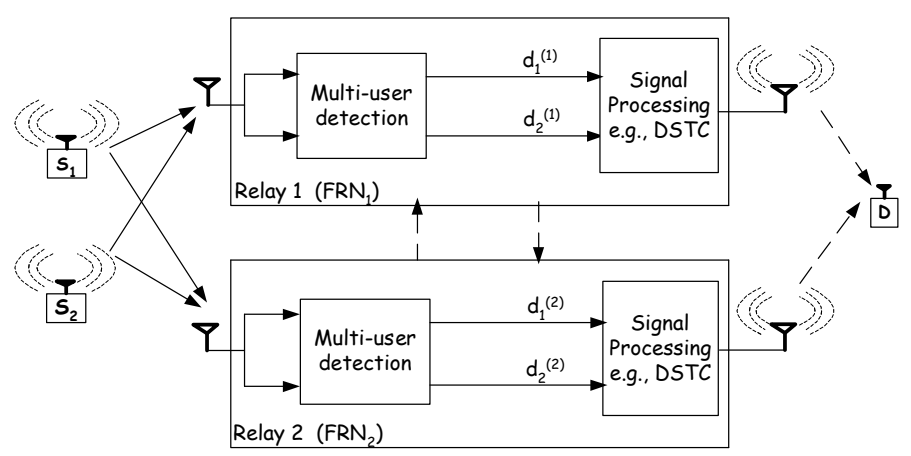

(a)

\begin{tabular}{|c|c|c|}
\hline $\begin{array}{l}s_{1}\left(d_{1}\right)-->F R N_{1}, F R N_{2} \\
j_{2}\left(d_{2}\right)-->F R N_{1}, F R N_{2}\end{array}$ & $\begin{array}{l}\mathrm{FRN}_{1}\left(\mathrm{~d}_{1}^{(1)}\right)-->>D \\
\mathrm{FRN}_{2}\left(\mathrm{~d}_{2}^{(2)}\right)-->D\end{array}$ & $\begin{array}{l}F R N_{1}\left(d_{2}{ }^{(1)}\right)-->D \\
F R N_{2}\left(d_{1}\left({ }^{(2)}\right)-\rightarrow D D\right.\end{array}$ \\
\hline
\end{tabular}

(b)

Fig. 2. Fixed relay-enabled user cooperation: Realization II.

TABLE I

THE ENCODING AND TRANSMISSION SEQUENCE FOR THE DISTRIBUTED SPACE TIME CODE

\begin{tabular}{||c|c|c||}
\hline \hline Relay station & FRN $_{1}$ transmits & FRN $_{2}$ transmits \\
\hline \hline time $t+T$ & $\hat{d}_{1}^{(1)}$ & $\hat{d}_{2}^{(2)}$ \\
\hline time $t+2 T$ & $-\hat{d}_{2}^{*(1)}$ & $\hat{d}_{1}^{*(2)}$ \\
\hline \hline
\end{tabular}

actions can be undertaken. First, the sum of the times allocated to the three slots can be made equal to that of the reference scheme. Second, a higher modulation constellation could be employed in the links to allow for the same bandwidth utilization with the reference scheme.

\section{AnAlysis of RelAy-ENABLED CoOperative COMMUNICATION SCHEMES}

We consider the source-relay links with each user carrying a single antenna and the fixed relay equipped with $L$ antennas. After matched filtering, sampling at symbol rate, the base band model for the received signal vector can be expressed as

$$
\mathbf{y}=\sum_{k=0}^{\mathcal{N}_{u}} \boldsymbol{\alpha}_{k} x_{k}+\mathbf{n}_{k}
$$

where $\boldsymbol{\alpha}_{k}$ is the propagation vector for the user $k$, and $\mathbf{n}_{k}$ is the corresponding IID noise. The vectors $\mathbf{y}, \boldsymbol{\alpha}_{k}, \mathbf{n}_{k}$ have a dimension $L$.

The task is to find a set of weight $\boldsymbol{\omega}$ (beamformer) that maximizes the SINR for the desired partner. We will denote this weight vector as $\boldsymbol{\omega}_{\text {opt }}$. However, for general $\boldsymbol{\omega}$, the power at the output of such a combiner can be expressed as

$$
P_{o}=P_{d}+P_{\mathrm{I}+\mathrm{N}}=E_{d}\left|\boldsymbol{\omega}^{*} \boldsymbol{\alpha}_{0}\right|^{2}+\boldsymbol{\omega}^{*} \mathbf{R}_{n n} \boldsymbol{\omega},
$$

where, $P_{d}$ is the desired user power, $P_{\mathrm{I}+\mathrm{N}}$ is the noise plus interferer power, and (*) is the conjugation transposition operation known as Hermitian operator and $\mathbf{R}_{n n}$ is the covariance matrix of the interference plus noise. 
The output SINR can represented as

$$
S I N R=\frac{P_{d}}{P_{\mathrm{I}+\mathrm{N}}}=\frac{E_{d}\left|\boldsymbol{\omega}^{*} \boldsymbol{\alpha}_{0}\right|^{2}}{\boldsymbol{\omega}^{*} \mathbf{R}_{n n} \boldsymbol{\omega}} .
$$

The optimum weight vector that yields the maximum SINR can be obtained by minimizing the denominator of (3). The minimization is subject to the constraint $\boldsymbol{\omega}^{*} \boldsymbol{\alpha}_{0}=a$. The Lagrange approach can be used to perform the minimization. Then, the maximum output SINR, simply denoted as $\gamma$ can be expressed as

$$
\gamma=E_{d} \frac{\left|\boldsymbol{\omega}_{\mathrm{opt}}^{*} \boldsymbol{\alpha}\right|^{2}}{\boldsymbol{\alpha}_{0}^{*} \mathbf{R}_{n n}^{-1} \boldsymbol{\alpha}_{0}}=E_{d} \frac{\left(\boldsymbol{\alpha}_{0}^{*} \mathbf{R}_{n n}^{-1} \boldsymbol{\alpha}_{0}\right)^{2}}{\boldsymbol{\alpha}_{0}^{*} \mathbf{R}_{n n}^{-1} \boldsymbol{\alpha}_{0}}=E_{d} \boldsymbol{\alpha}_{0}^{*} \mathbf{R}_{n n}^{-1} \boldsymbol{\alpha}_{0}
$$

and $\mathbf{R}_{n n}$ is obtained as

$$
\begin{aligned}
\mathbf{R}_{n n} & =E\left[\left(\mathbf{y}-\boldsymbol{\alpha}_{0} x_{0}\right)\left(\mathbf{y}-\boldsymbol{\alpha}_{0} x_{0}\right)^{*}\right] \\
& =E_{I} \sum_{k=1}^{\mathcal{N}_{u}} E\left[\boldsymbol{\alpha}_{k} \boldsymbol{\alpha}_{k}^{*}\right]+\sigma^{2} \mathbf{I}_{L}=\mathbf{R}_{I}+\mathbf{R}_{n}
\end{aligned}
$$

This covariance matrix represents an average performed over a period shorter that the reciprocal of the fading rate. Equal energy signals (MPSK modulation schemes) are considered.

The cooperative scheme identifies two users that are to be engaged in cooperation by the relays. Some simplifying approaches are worth considering. One approach is to consider that at each relay, one strong user (among the $\mathcal{N}_{u}$ users) is interfering and others can be lumped with the system noise.

For the multi-antenna relays that are investigated here, $L$ is greater than the number of interfering (or dominant) users. In this scenario the distribution of the interference covariance matrix $\mathbf{R}_{I}$ is pseudo-Wishart's [5]. Hence, $\mathbf{R}_{I}$ has a single (dominant) non-zero eigen-value $\phi_{1}$ which is real and positive. The remaining $\phi_{i}, i=2, \cdots, L$ are zeros. Thus, the PDF of $\phi_{1}$ of $\mathbf{R}_{I}$ can be expressed using the joint PDF of pseudoWishart distribution, which can be simplified to

$$
p\left(\phi_{1}\right)=\frac{\phi_{1}^{L-1}}{(L-1) !} \exp \left(-\phi_{1}\right), \phi_{1}>0 .
$$

Corresponding to each $\phi_{i}$ is an eigen-vector $\boldsymbol{v}_{i}$. That is $\mathbf{R}_{I}=\sum_{i=1}^{L} \phi_{i} \boldsymbol{v} \boldsymbol{v}^{*}$, where $\phi_{i}=0 \mid i>1$. Note that the $\boldsymbol{v}^{\prime} \mathrm{s}$ are orthonormal eigenvectors of the interference correlation matrix. Hence, $\gamma$ in (4) can be expressed as [5], [6]

$$
\gamma=\frac{E_{d}\left|\boldsymbol{\alpha}_{0}^{*} \boldsymbol{v}_{1}\right|^{2}}{E_{I} \phi_{1}+N_{0}}+\sum_{i=2}^{L} \frac{E_{d}\left|\boldsymbol{\alpha}_{0}^{*} \boldsymbol{v}_{i}\right|^{2}}{N_{0}}
$$

which implies that the conditional characteristic function can written as

$$
\begin{aligned}
\varphi_{\gamma \mid \phi_{1}}(j v) & =\frac{1}{\left(1-j v E_{d} / N_{0}\right)^{L-1}} \times \frac{1}{\left(1-j v \frac{E_{d}}{E_{I} \phi_{1}+N_{0}}\right)} \\
& =\frac{1}{\left(1-j v \bar{\gamma}_{1}\right)^{L-1}} \times \frac{1}{\left(1-j v \bar{\gamma}_{2}\right)}
\end{aligned}
$$

where $\bar{\gamma}_{1}=\frac{E_{d}}{N_{0}}$ and $\bar{\gamma}_{2}=\frac{E_{d}}{E_{I} \phi_{1}+N_{0}}$.

The PDF of $\gamma$ conditioned on $\phi_{1}$ can be obtained by taking the inverse Fourier transform of (8). This can be achieved by resolving this equation into its partial fractions, thus, leading to the following PDF:

$$
p\left(\gamma \mid \phi_{1}\right)=\sum_{i=1}^{2} \sum_{t=1}^{\mathcal{L}_{i}} \zeta_{i, t} \frac{\gamma^{t-1}}{\bar{\gamma}_{i}^{t} \Gamma[t]} \exp \left(-\frac{\gamma}{\bar{\gamma}_{i}}\right)
$$

where $\mathcal{L}_{1}=L-1, \mathcal{L}_{2}=1$ and

$\zeta_{i, t}=\left.\frac{1}{\left(\mathcal{L}_{i}-t\right) !\left(-\bar{\gamma}_{i}\right)^{\mathcal{L}_{i}-t}} \frac{\partial^{\mathcal{L}_{i}-t}}{\partial \omega^{\mathcal{L}_{i}-t}}\left(1-\bar{\gamma}_{i} \omega\right)^{\mathcal{L}_{i}} \varphi_{\gamma \mid \phi}(\omega)\right|_{\omega=\frac{1}{\bar{\gamma}_{i}}}$.

Error probability analysis can be undertaken with this PDF of the output SINR. For an illustration, the symbol error probability or rate (SER) for MPSK conditioned on this SINR is given by

$$
P_{\operatorname{mpsk}}\left(e \mid \gamma, \phi_{1}\right) \approx h \operatorname{erfc}(\sqrt{\gamma \lambda}),
$$

where $\lambda=q \sin ^{2}\left(\frac{\pi}{M}\right)$. For BPSK $M=2, h=1 / 2, q=1$, and equality is satisfied, and for higher MPSK constellations $h=1$.

The unconditional error performance can be obtained by averaging (10) over the PDFs of $\gamma$ and $\phi_{1}$. Finally, the SER can be written as

$$
\begin{aligned}
P_{e} & =\int_{0}^{\infty} \int_{0}^{\infty} P_{\mathrm{mpsk}}\left(e \mid \gamma, \phi_{1}\right) p\left(\gamma \mid \phi_{1}\right) p\left(\phi_{1}\right) d \gamma d \phi_{1}(11) \\
& =\int_{0}^{\infty} p\left(\phi_{1}\right) \int_{0}^{\infty} P_{\mathrm{mpsk}}\left(e \mid \gamma, \phi_{1}\right) p\left(\gamma \mid \phi_{1}\right) d \gamma d \phi_{1} .
\end{aligned}
$$

The integral $\int_{0}^{\infty} P_{\text {mpsk }}\left(e \mid \gamma, \phi_{1}\right) p\left(\gamma \mid \phi_{1}\right) d \gamma$, which is the error performance conditioned on the eigen-value $\phi_{1}$, can be evaluated using the PDF in (9). This integral is denoted as $P\left(e \mid \phi_{1}\right)$. Thus, $P\left(e \mid \phi_{1}\right)$ can be expressed as

$$
P\left(e \mid \phi_{1}\right)=\sum_{i=1}^{2} \sum_{t=1}^{\mathcal{L}_{i}} \zeta_{i, t} \frac{h \Gamma[t+1 / 2]}{\sqrt{\pi} \Gamma[t]} B_{x_{i}}[t, 1 / 2],
$$

where $x_{i}=\frac{1}{\bar{\gamma}_{i}+1}$ and $B_{x}[\cdot, \cdot]$ is the incomplete beta function.

The SER at the relay for a given user is obtained as

$$
P_{e} \simeq \int_{0}^{\infty} P\left(e \mid \phi_{1}\right) p\left(\phi_{1}\right) d \phi_{1} .
$$

The integral in (13) can be evaluated using numerical means.

The formulation of the E2E error performance assumes that each relay experiences independent error rates and that any error committed by the cooperating relay results in an error being committed at the destination. The actual system performance could then be better than the results that are estimated using these assumptions. The reason is that it is possible for an error to occur at the relays and these erroneous bits result in correct bit decisions at the destinations. The E2E error rate can be expressed as

$$
P_{e, \mathrm{E} 2 \mathrm{E}}=1-\prod_{i=1}^{2}\left(1-P_{e, i}\right),
$$

where in the two-hop scenario, $P_{e, 1}$ is the error rate at the first hop (relay) and $P_{e, 2}$ is the error rate at the destination (the second hop). 


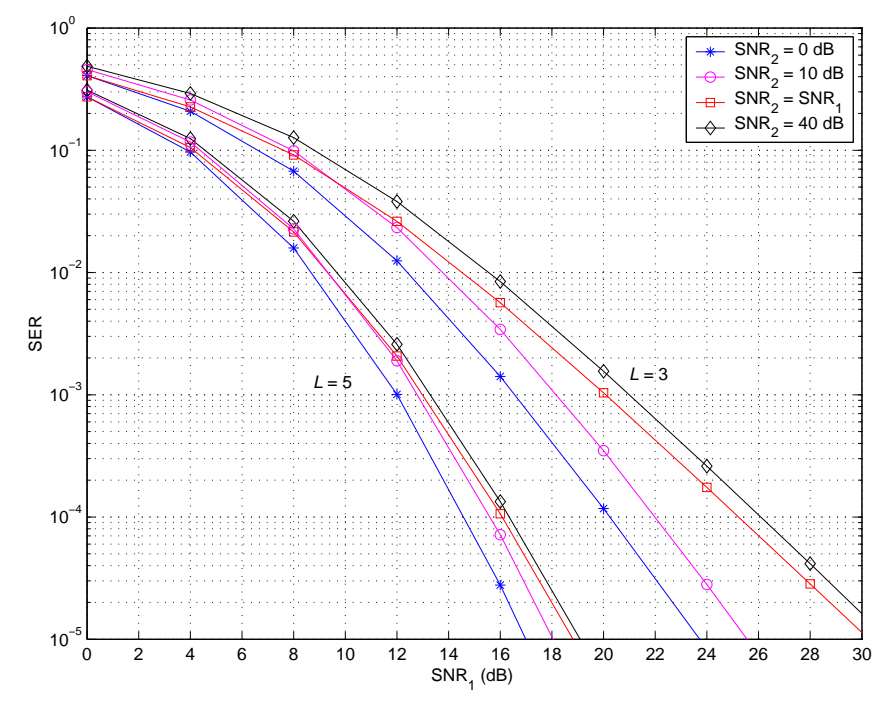

Fig. 3. The SER of SDMA relay in Rayleigh fading channels for the desired user for different power levels of partner $(L=3$ and $L=5), 8$-PSK.

\section{A. Error Rate for Transmit Diversity}

The two transmit diversity described [4] offers an error rate that is exactly the same with that of receive diversity. This implies that with proper power adjustment, the performance of the transmit diversity schemes $(2 \times 1$ and $2 \times 2)$ can be evaluated using those of the corresponding receive diversity schemes [7]. Therefore, for these transmit schemes the SER expression given as

$$
P_{e, 2}=\frac{h \Gamma[L+1 / 2]}{\sqrt{\pi} \Gamma[L]} B_{\mu}[L, 1 / 2]
$$

can be used, where $\mu=\frac{1}{\lambda \bar{\gamma}+1}$ and $\bar{\gamma}$ is the adjusted SNR. Note that $L=2$ for $(2 \times 1)$ and $L=4$ for $(2 \times 2)$ transmit schemes.

\section{NUMERICAL EXAMPLES}

Fig. 3 shows the error performance of a relay for $L=3$ and $L=5$ with 8-PSK modulation. Each user has single antenna and as well as the destination. However, the destination implements Alamouti-type receiver. We observe that a diversity order of approximately 2.0 (for $L=3$ ) and 3.9 (for $L=5$ ) can be computed from the curves representing cases for $\mathrm{SNR}_{2}=40 \mathrm{~dB}$. In general, for infinite power interferer the relay performance is bounded by the performance of $L-1$ diversity system with no interferer in agreement with [8].

Fig. 4 shows E2E BER for realization I. For $L=2$ the new scheme provides each of the two users in the network with an error rate that is not inferior to a single user network. In both networks, 8-PSK modulation format is adopted. This error performance advantage comes at the expense of $33 \%$ loss in spectral efficiency. The situation is different for large $L$. For instance, when $L=3$, the performance of each user in the new scheme is superior to that of the single user network using BPSK. This represents both diversity and spectral (multiplexing) gains. Considering the overall network, an improvement in spectral efficiency of $100 \%$ is achieved with the new scheme in addition to the large SNR gain at a low

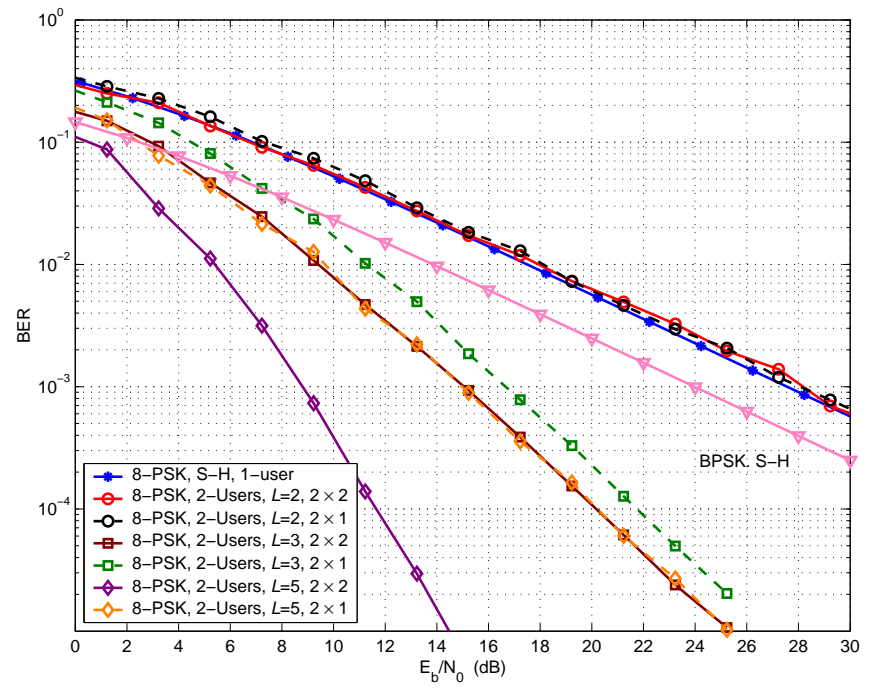

Fig. 4. The E2E BER of realization I in Rayleigh fading channels.

error performance. The new scheme achieves an SNR gain as large as $7.5 \mathrm{~dB}$ (for $L=3$ ) and $9 \mathrm{~dB}(L=5)$ over the BPSK at a BER of $10^{-3}$ (Fig. 4). The margin of the gain is larger in low BER regime. Furthermore, the relay-enabled user cooperation scheme can provide each cooperating user with a performance that approaches that of a single user network implementing 2 $x 1$ Alamouti scheme. The scheme using SDMA requires only five antennas at the fixed relays to achieve this performance. It should be noted that each user terminal has one antenna.

The BER is also shown for two receive antennas at the destination. In this case, each antenna of the two (distributed) relays and the two antennas of the destination form a virtual 2 x 2 antenna scheme. A significant performance improvement due to the addition of one antenna at the destination is observed. For instance, at BER of $10^{-4}$, the virtual array $2 \times 2$ of the second hop is superior to its $2 \times 1$ counterparts by about $8 \mathrm{~dB}$.

Fig. 5 shows the system performance when the power of the cooperating partner is set to $0,10,40 \mathrm{~dB}$ and $\mathrm{SNR}_{2}=\mathrm{SNR}_{1}$. The destination is equipped with one antenna. It is observed that for $0 \mathrm{~dB}$, the E2E diversity order obtained for each partner is about 2. However, for $\mathrm{SNR}_{2}=10 \mathrm{~dB}$ this diversity order is slightly reduced. When $\mathrm{SNR}_{2}$ increases to $40 \mathrm{~dB}$ the overall diversity order drops to 1 .

A performance comparison for the simulated and analytical results is presented in Fig. 6. The analytical results are the solid curves while simulations are denoted with asterisks. The analytical results assume that the partner comes with a $40 \mathrm{~dB}$ power while the simulation results are for infinite power interferer. The curves demonstrate that analytical results, though approximations provide a reasonably close system performance. It is important to note that the number of antenna at the relay has been used to improve the S-R channels (i.e., the first hop). This explains why the diversity order is restricted to the number of relay nodes. Had all the antennas been engaged in the transmission in the second hop, it would have been possible to increase the diversity order. 


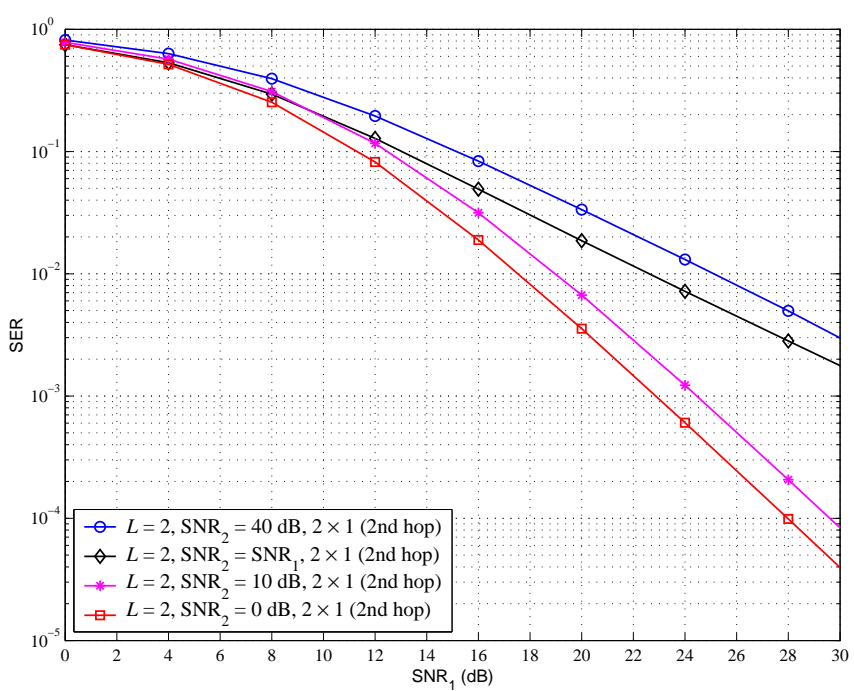

Fig. 5. The E2E SER for different power levels of partner ( $L=2$, 8-PSK).

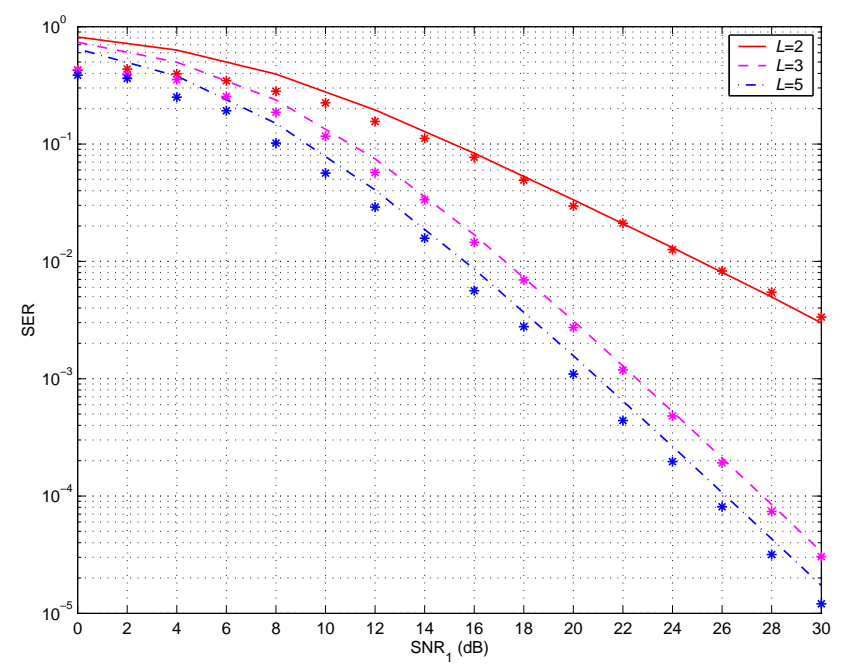

Fig. 6. The simulated E2E SER for infinite power of partner $\mathrm{SNR}_{2}=\infty$ $\mathrm{dB}$ and the analytical SER for $\mathrm{SNR}_{2}=40 \mathrm{~dB}(L=2,3,5,8-\mathrm{PSK})$.

Another important point that can be deduced from these results is that in the multi-antenna multi-relay scheme, the minimum number of diversity order required at the relay to achieve a E2E diversity order equal to the number of cooperating relays is two. This is the reason why for $L=3$, the diversity order obtained is the same as for all interfering power.

Fig. 7 provides the error performance for realization II. It is observed that when the first hop detection is made reliable (through CRC/ARQ protection) a diversity order of two is obtained for each of the users in the new scheme. In this study, suboptimal low complexity single-user detection is performed. At high SNR the spectral loss in realization II due to ARQ is minimal. Due to space constraint the figure is omitted but we derive the following discussions from it. Simulation results indicate that for SNR as low as $10 \mathrm{~dB}$, the asymmetric networks (where the relays are located midway between the users and destination) request for repeat made is below $3 \%$ of the time while for the symmetric networks (distance dependent attenuation is de-emphasized) it is about

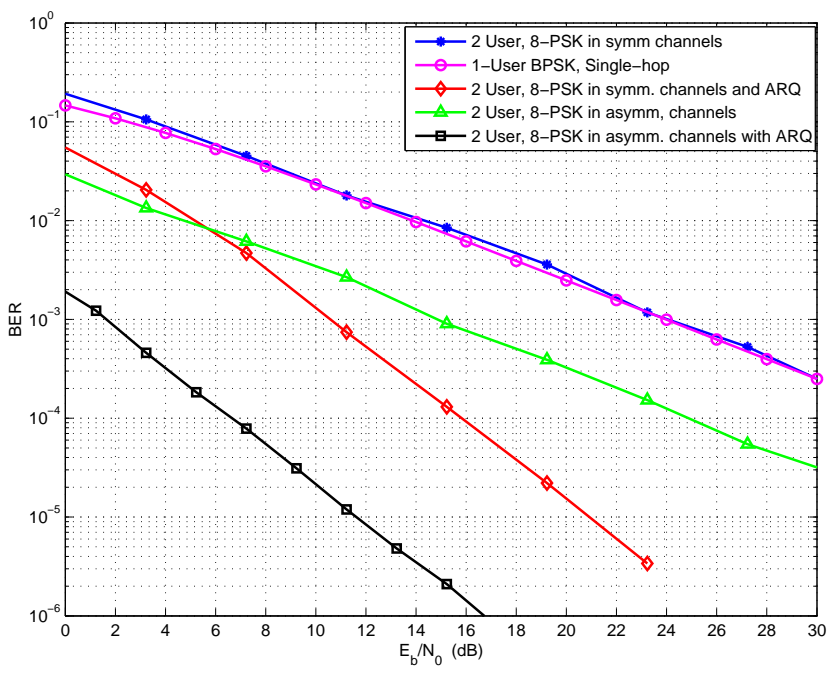

Fig. 7. Performance of relay-enabled user cooperation using realization II.

$29 \%$ of the time. At SNR above $15 \mathrm{~dB}$, the percentage drops to less than $1 \%$ for asymmetric networks and less than $10 \%$ for the symmetric networks. At sufficiently high SNR the system is less dependent on the ARQ demonstrating minimal impact on the system throughput.

\section{CONCLUSION}

As an add-on to network infrastructure-based fixed relays, this work investigates the cooperation of these relays to acquire diversity and multiplexing gains. Since the cooperation is transparent to the users (i.e. users are ignorant of it) the proposed schemes present a practical realization for user cooperation schemes as it eases the concerns about security and privacy and incentive for terminals to assist others. In addition, BER and spectral efficiencies obtained are superior to the referenced single-hop transmissions. Finally, the schemes can be used in any network since current terminals do not require any modifications. Instead, the terminal complexity/cost are transferred to the relay level. It will be more economical to increase the cost of few relay stations rather than the cost of modifying thousands of terminals.

\section{REFERENCES}

[1] J. Laneman, D. Tse, and G. Wornell, "Cooperative diversity in wireless networks: efficient protocols and outage behavior,' IEEE Transactions on Information Theory, 50(11), pp. 3062-3080, Dec. 2004.

[2] A. Sendonaris, E. Erkip, and B. Aazhang "User cooperation diversity - Part I: system description," IEEE Transactions on Communications, 51(11), pp. 1927-1938, Nov. 2003.

[3] H. Hu, H. Yanikomeroglu, D. Falconer, and S. Periyalwar, "Range extension without capacity penalty in cellular networks with digital fixed relays", IEEE Globecom, 2004.

[4] S. Alamouti, "A simple transmit diversity technique for wireless communication," IEEE Journal Selected Areas in Communications, 16(8), pp. 1451-1458, Oct. 1998.

[5] R. Mallik, M. Win, M. Chiani, and A. Zanella, "Bit-error probability for optimum combining of binary signals in the presence of interference and noise," IEEE Transactions on Wireless Communications, 3(2), pp. 395-407 Mar. 2004.

[6] H. Van Trees, Optimum Array Processing, Wiley, 2002.

[7] A. Adinoyi and H. Yanikomeroglu, "Cooperative relaying in multiantenna fixed relay networks", to appear in IEEE Transactions on Wireless Communications, 2007.

[8] J. Winters, "Optimum combining in digital mobile radio with cochannel interference," IEEE Journal on Selected Areas in Communications, no. 4, pp. 528-539, July 1984. 